\title{
Improved Characterization of Steel Samples by SEM/EDS Through the Use of a Silicon Drift Detector
}

\author{
John Konopka
}

Thermo Fisher Scientific, Madison, WI, USA

The Silicon Drift Detector (SDD) was first conceived of about 30 years ago and has largely supplanted the $\mathrm{Si}(\mathrm{Li})$ detector[1]. The current generation of commercially available SDD meets or exceeds the performance of a $\mathrm{Si}(\mathrm{Li})$ detector in nearly every way. The purpose of this work is to illustrate how an SDD applied to the analysis of a set of steel samples provides more detailed and reliable information in roughly the time it took to collect a single spectrum with a $\mathrm{Si}(\mathrm{Li})$ detector.

The samples are three steels from a set of Brammer standards; SS 303, SS 410 and SS 430. Steels are difficult to analyze because they are often inhomogeneous. They can consist of multiple phases and may contain inclusions. The bulk composition cannot be inferred from a single point analysis. This work tests analytical methods that provide more detailed and reliable characterization of these complex materials.

The samples, as received, are flat and polished so no sample preparation was required besides cleaning.

With the $\mathrm{Si}(\mathrm{Li})$ detector, a spectrum was typically acquired at about $2,000 \mathrm{cps}$ storage rate and $30 \%$ dead time for about 100 - 200s live time or clock time[2][3]. The SDD counts X-rays more quickly under the same conditions than does a $\mathrm{Si}(\mathrm{Li})$ detector and can count even more quickly if the beam current can be increased[4]. Moreover, SDDs are available providing much more solid angle while maintaining approximately the same resolution. This allows high speed acquisition with less beam current which provides higher spatial resolution when using a tungsten gun SEM and opens this analysis to a wider variety of SEMs, not all of which can produce high beam currents.

Table 1 compares the sensitivity of two different SDDs in counts per second per nanoAmp. Note the much higher sensitivity of the larger detector which allows fast acquisition at very low beam currents.

It is currently possible to collect at least sixteen times as many spectra in a given time as previously while collecting even more counts in each spectrum. Table 2 shows results for an application of this speed using a sample of SS 303. Sixteen spectra were acquired in a grid pattern on the sample for a total of $\sim 150$ s live time. In the first case the positions of each spot were chosen to only analyze the matrix. The second spot locations were selected without regard to the presence of phases or inclusions. The larger standard deviation of the results from second set of measurements should be a warning flag to use care in interpreting these results. In this example the inclusions were visible in the BSE image. This is not always the case. This method takes little time but is a reliable test for the homogeneity of a sample regardless of whether variations in composition are visible in the electron image. This helps the analyst avoid making claims based on a single spot analysis which may not be representative of the sample.

A second use for the SDD's high throughput is to search for trace constituents. As has been pointed out 
elsewhere, collecting vastly more counts makes visible trace constituents[4]. Spending a little more time or raising the beam current allows for collecting many millions of counts in the spectrum revealing trace constituents present at less than $0.5 \%$ by weight, if they are not overlapped by a major peak. The presence or absence of trace elements can assist in the identification of steel particles.

Acquiring many spectra at one location provides a time series that reveals the accumulation of contaminants as a function of time. Acquiring a number of spectra in a region provides a statistical view of the uniformity of the composition of the sample in that region. Acquiring a single spectrum for longer time can reveal trace constituents. All of these can be performed today in roughly the time it used to require to collect a single spectrum. This should become the normal way of analyzing materials.

[1] Gatti E., and P. Rehak, Nucl. Instrum. Methods A, 1984 42, 12.

[2] C. E. Fiori, C. R. Swyt, Microbeam Analysis, 1992, 89-95

[3] I. R. Harrowfield, C. M. MacRae, Microbeam Analysis, 1988, 267-270

[4] D. E. Newbury, N. W. M. Ritchie, Materials Science and Technology, 2016, 1017-1023

\begin{tabular}{|c|c|}
\hline Area & counts / sec / nA \\
\hline $\mathbf{3 0} \mathbf{m m}^{\mathbf{2}}$ & 3,290 \\
\hline $\mathbf{1 0 0} \mathbf{~ m m}^{\mathbf{2}}$ & 46,266 \\
\hline
\end{tabular}

Table 1. Fe K line intensity measured from SS 410 on two different SDDs. The larger detector gains about 3x sensitivity in size and about $4 \mathrm{x}$ sensitivity because the shape allows it to be much closer to the sample. Also, it is a window-less detector.

\begin{tabular}{|c|c|c|c|c|}
\hline \multicolumn{2}{|c}{} & \multicolumn{2}{c}{ matrix } & \multicolumn{2}{c|}{ without regard to inclusions } \\
\hline Ovg & & Std Dev & Avg & Std Dev \\
\hline Si & 0.57 & 0.05 & 0.18 & 0.72 \\
\hline S & & & 1.11 & 0.08 \\
\hline Cr & 18.23 & 0.21 & 17.81 & 2.80 \\
\hline Mn & 2.15 & 0.23 & 4.58 & 1.13 \\
\hline Fe & 71.07 & 0.55 & 67.92 & 6.93 \\
\hline Ni & 7.52 & 0.54 & 7.39 & 0.56 \\
\hline Mo & 0.48 & 0.06 & 0.33 & 0.18 \\
\hline
\end{tabular}

Table 2. Statistics of two sets of 16 measurements, $8 \mathrm{~s}$ live time each, from SS 303. For each measurement the beam was scanned over an area $\sim 10$ microns in diameter. Note the much larger standard deviations when inclusions could be included in the scanned area. 